\title{
Design of Reliable Controllers for Symmetric Composite Systems: Primary Contingency Case
}

\author{
Guang-Hong Yang, Jinaliang Wang and Cheong Boon Soh \\ School of EEE, Nanyang Technological University, Singapore 639798 \\ egyang@ntuvax.ntu.ac.sg, ejlwang@ntuvax.ntu.ac.sg, chsoh@wj7.eee.ntu.ac.sg \\ James Lam \\ Department of ME, The University ooooof Hong Kong, Pokfulam Road, Hong Kong \\ jlam@hkuxa.hku.hk
}

\begin{abstract}
This paper discusses the reliable controller design problem for symmetric composite systems composed of several identical subsystems. A reliable controller design procedure is presented in terms of the solutions to the Algebraic Riccati Equations. The order of these AREs is much lower than that of the symmetric composite system. The resulting closed-loop system is reliable in that it provide guaranteed internal stability and $H_{\infty}$ performance when all sensors and actuators are operational as well as when the sensors or actuators of a prescribed subsystem experiences an outage.
\end{abstract}

\section{Introduction}

Symmetric composite systems are systems consisting of identical subsystems with interconnections described by constant block-symmetric matrices. This class of systems occurs in very diverse areas such as electric power systems, industrial manipulators, computer networks, etc. [1] [2] [6] [8]. In recent years there has been a great interest in symmetric composite systems. Lunze first proposed the state model of symmetric composite systems, and investigated some of the fundamental properties of such systems [1]. In [6], a synthesis procedure for decentralized controllers for symmetric compositc systems is presented and important characteristics of such systems are observed. For uncertain symmetric composite systems, a robust controller design procedure is given in [8].

Recently, some approaches to the design of reliable controllers that retain stability and $H_{\infty}$ performance have been developed by several authors [3] - [5] [7]. In [7], Veillette $e t$ al presented a methodology for the design of reliable centralized and decentralized control systems by using the Algebraic Riccati Equation (ARE) approach The resulting controller guarantees closed-loop internal stability and $H_{\infty}$ performance not only when

0-7803-3590-2/96 \$5.00 @ 1996 IEEE all control components are operational, but also in case of some admissible control component outages. In [3], Medanic investigated the single contingency reliable design problem, and presented a technique for $H_{\infty}$-norm bounding design that results in performance reliable with respect to the outage of any one sensor or any one actuator by introducing a redundant sensor and a redundant actuator.

In this paper, we study the reliable control problem for symmetric composite systems. By making use of the symmetric structures, the order of the AREs involved in the design process is drastically reduced. The paper is organized as follows. The mathematical description of symmetric composite systems and and problem formulation are given in Section 2, together with some technical preliminaries. In Section 3 a reliable controller design procedure is presented in terms of the solutions to the Algebraic Riccati Equations (AREs) whose order is much lower than that of the system. The controller is reliable with respect to the outage of sensors or actuators of a prescribed subsystem. Finally, some concluding remarks are given in Section 4.

\section{Problem formulation and preliminaries}

The symmetric composite system $\boldsymbol{\Sigma}$ under consideration consists of $N(N>1)$ identical subsystems and the overall system is described by composite equations of the following form

$$
\begin{aligned}
\Sigma: \quad \dot{x} & =A x+B u+G w \\
y & =C x+w_{0} \\
z & =\left[\begin{array}{c}
H x \\
u
\end{array}\right]
\end{aligned}
$$

where for $i=1, \cdots, N$,

$$
x=\left[x_{1}^{T}, \cdots, x_{N}^{T}\right]^{T}, \quad x_{i} \in \mathbf{R}^{n}
$$




$$
\begin{aligned}
u & =\left[u_{1}^{T}, \cdots, u_{N}^{T}\right]^{T}, \quad u_{i} \in \mathbf{R}^{m} \\
y & =\left[y_{1}^{T}, \cdots, y_{N}^{T}\right]^{T}, \quad y_{i} \in \mathbf{R}^{p}
\end{aligned}
$$

Here $u_{i}, x_{i}$, and $y_{i}$ are, respectively, the input, state, and output of the $i$ th subsystem; $z \in \mathbf{R}^{r N+m N}$ is the output to be regulated; and $w$ and $w_{0}$ are the squareintegrable disturbances. The composite matrices $A \in$ $\mathbf{R}^{n N \times n N}, B \in \mathbf{R}^{n N \times m N}, C \in \mathbf{R}^{p N \times n N}, G \in \mathbf{R}^{n N \times q N}$ and $H \in \mathbf{R}^{r N \times n N}$ all have block-symmetric structures. For example,

$$
A=\left[\begin{array}{cccc}
A_{11} & A_{12} & \cdots & A_{12} \\
A_{12} & A_{11} & \cdots & A_{12} \\
\vdots & \vdots & \ddots & \vdots \\
A_{12} & A_{12} & \cdots & A_{11}
\end{array}\right]
$$

When an actuator outage occurrs in, say, the first subsystem, the $B$ matrix in (1) is replaced by

$$
B_{0}=\left[\begin{array}{cccc}
0 & B_{12} & \cdots & B_{12} \\
0 & B_{11} & \cdots & B_{12} \\
\vdots & \vdots & \ddots & \vdots \\
0 & B_{12} & \cdots & B_{11}
\end{array}\right]
$$

Similarly, when a sensor outage occurrs in, say, the first subsystem, the $C$ matrix in (2) is replaced by

$$
C_{0}=\left[\begin{array}{cccc}
0 & 0 & \cdots & 0 \\
C_{12} & C_{11} & \cdots & C_{12} \\
\vdots & \vdots & \ddots & \vdots \\
C_{12} & C_{12} & \cdots & C_{11}
\end{array}\right]
$$

By defining

$$
\begin{aligned}
B_{1}= & {\left[\begin{array}{cccc}
B_{11} & 0 & \cdots & 0 \\
B_{12} & 0 & \cdots & 0 \\
\vdots & \vdots & \ddots & \vdots \\
B_{12} & 0 & \cdots & 0
\end{array}\right] } \\
C_{1}= & {\left[\begin{array}{cccc}
C_{11} & C_{12} & \cdots & C_{12} \\
0 & 0 & \cdots & 0 \\
\vdots & \vdots & \ddots & \vdots \\
0 & 0 & \cdots & 0
\end{array}\right] }
\end{aligned}
$$

we have the following decompositions for the $B$ and $C$ matrices.

$$
B=B_{0}+B_{1}, \quad C=C_{0}+C_{1}
$$

Motivated by the controller structure by Veillette et al. in [7], we consider dynamic controllers of the following form.

$$
\begin{aligned}
\dot{\xi} & =(A+B K) \xi+G_{+} \hat{w}+L(y-C \xi) \\
u & =K \xi, \quad \xi \in \mathbf{R}^{n N}
\end{aligned}
$$

where $\operatorname{dim} \hat{w}=\operatorname{dim} w+\operatorname{dim} u$. Then the reliable control problem considered in this paper is defined as follows. Given the symmetric composite linearsystem $\Sigma$ in (1) - (3), design a controller of the form (10)-(11) such that the resulting closed-loop system is asymptotically stable, and the $H_{\infty}$-norm of the closed-loop transfer function matrix from $\left[w^{T}, w_{0}^{T}\right]^{T}$ to $z$ is bounded by some prescribed $\gamma>0$ when all sensors and actuators are operational as well as when the sensors and actuators of one, but only one, prescribed subsystem experience outages.

Due to the symmetry in the system, it is sufficent to consider only outages in the first subsystem. Before going to the next section to give the design procedure for the above reliable control problem, the following technical preliminaries are needed.

Suppose that $v_{0}, \cdots, v_{s-1}$ are the $s$ roots of unity in complex plane. That is $v_{j}=\exp (2 \pi j \sqrt{-1} / s), j=$ $0,1, \cdots, s-1$. Let $m_{j}=\left[1, v_{j}, v_{j}^{2}, \cdots, v_{j}^{s-1}\right]^{T}$. For given positive integers $p, q$ and $s$, denote

$$
\lambda=\left\{\begin{array}{cc}
\frac{s-1}{2} & \text { for } s \text { odd } \\
\frac{s}{2} & \text { for } s \text { even }
\end{array}\right.
$$

and

$$
T_{R}(p, q, s)=\operatorname{diag}\left[I_{p}, R_{s} \otimes I_{q}\right]
$$

where $I_{q}$ denotes the $q \times q$ identity matrix, $\otimes$ denotes the Kronecker product and the matrix $R_{s}$ is defined as follows

$$
R_{s}=M_{s} U_{s}
$$

with

$$
\begin{gathered}
M_{s}=\left\{\begin{array}{cc}
\frac{1}{\sqrt{s}}\left[m_{0} m_{1} m_{N-1} m_{2} m_{N-2} \cdots m_{\lambda} m_{s-\lambda}\right] \\
\frac{1}{\sqrt{s}}\left[m_{0} m_{1} m_{N-1} m_{2} m_{N-2} \cdots\right. \\
\left.m_{\lambda-1} m_{\lambda} m_{s-\lambda+1}\right] & \text { for } s \text { odd }
\end{array}\right. \\
U_{s}=\left\{\begin{array}{cc}
\operatorname{diag}[1, \underbrace{V, \cdots, V]}_{\lambda} & \text { for } s \text { odd } \\
\operatorname{diag}[1, \underbrace{V, \cdots, V,}_{\lambda-1} 1] & \text { for } s \text { even }
\end{array}\right.
\end{gathered}
$$

and

$$
V=\frac{1}{\sqrt{2}}\left[\begin{array}{cc}
1 & \sqrt{-1} \\
1 & -\sqrt{-1}
\end{array}\right]
$$

It is straightforward to verify that $R_{s}$ is a real orthogonal matrix. (In fact, $R_{s}$ is also normal.) Hence, $T_{R}(p, q, s)$ is a real orthogonal matrix.

Lemma 1: For any given matrices $E_{00} \in \mathbf{R}^{q_{0} \times k_{0}}$, $E_{01} \in \mathbf{R}^{q_{0} \times k}, E_{10} \in \mathbf{R}^{q \times k_{0}}, E_{11} \in \mathbf{R}^{q \times k}$ and $E_{11} \in$ 


$$
\begin{aligned}
& \mathbf{R}^{q \times k}, \text { denote } \\
& E=\left[\begin{array}{ccccc}
E_{00} & E_{01} & \cdots & E_{01} & E_{01} \\
E_{10} & E_{11} & \cdots & E_{12} & E_{12} \\
\vdots & \vdots & \ddots & \vdots & \vdots \\
E_{10} & E_{12} & \cdots & E_{11} & E_{12} \\
E_{10} & E_{12} & \cdots & E_{12} & E_{11}
\end{array}\right] \in \mathbf{R}^{\left(q_{0}+s q\right) \times\left(k_{0}+s k\right)} \\
& E_{p}=\left[\begin{array}{cc}
E_{00} & \\
\sqrt{s} E_{10} & E_{11}+(s-1) E_{12}
\end{array}\right] \\
& E_{m}=\left[\begin{array}{c}
E_{11}-E_{12} \\
E_{01}
\end{array}\right.
\end{aligned}
$$

Then

$$
T_{R}^{T}\left(q_{0}, q, s\right) E T_{R}\left(k_{0}, k, s\right)=\operatorname{diag}\left[E_{p}, E_{m}, \cdots, E_{m}\right]
$$

Proof: Note that

$$
\begin{aligned}
1+v_{j}+v_{j}^{2}+\cdots+v_{j}^{s-1} & = \begin{cases}0 & j=1, \cdots, s-1 \\
1 & j=s\end{cases} \\
\sum_{i=0}^{s-1}\left(\bar{v}_{j} v_{k}\right)^{i} & = \begin{cases}0 & j \neq k \\
s & j=k\end{cases}
\end{aligned}
$$

where $\bar{v}_{j}$ is the complex conjugate of $v_{j}$ Denoting by $E_{1}$ the matrix $E$ with the $1^{\text {st }}$ block row and the $1^{\text {st }}$ block column deleted, and $(.)^{H}$ the Hermitian transpose of a matrix, we have, for $s$ odd

$$
\left(\operatorname{diag}\left[I_{q_{0}} \quad M_{s} \otimes I_{q}\right]\right)^{H} E
$$$$
=\left[\begin{array}{c|c}
E_{00} & E_{01} E_{01} \cdots E_{01} \\
\hline \sqrt{s} E_{10} & \\
\frac{1}{\sqrt{s}} \sum_{i=0}^{s-1} \bar{v}_{1}^{i} E_{10} & \left(M_{s} \otimes I_{q}\right)^{H} E_{1} \\
\vdots & \\
\frac{1}{\sqrt{s}} \sum_{i=0}^{s-1} \bar{v}_{s-\lambda}^{i} E_{10} &
\end{array}\right]
$$

$$
=\left[\begin{array}{c|c}
E_{00} & E_{01} E_{01} \cdots E_{01} \\
\hline \sqrt{s} E_{10} & \\
0 & \left(M_{s} \otimes I_{q}\right)^{H} E_{1} \\
\vdots &
\end{array}\right]
$$

But

$$
\left(M_{s} \otimes I_{q}\right)^{H} E_{1}
$$

$$
=\left(M_{s} \otimes I_{q}\right)^{H}\left[\begin{array}{ccccc}
E_{11} & E_{12} & \cdots & E_{12} & E_{12} \\
E_{12} & E_{11} & \cdots & E_{12} & E_{12} \\
\vdots & \vdots & \ddots & \vdots & \vdots \\
E_{12} & E_{12} & \cdots & E_{11} & E_{12} \\
E_{12} & E_{12} & \cdots & E_{12} & E_{11}
\end{array}\right]
$$

who's $j^{\text {th }}$ column (for $j=1, \cdots, s$ ) is

$$
\frac{1}{\sqrt{s}}\left[\begin{array}{c}
E_{11}+(s-1) E_{12} \\
\bar{v}_{1}^{j-1} E_{11}+\left(\sum_{i=0, i \neq j-1}^{s-1} \bar{v}_{1}^{i}\right) E_{12} \\
\bar{v}_{s-1}^{j-1} E_{11}+\left(\sum_{i=0, i \neq j-1}^{s-1} \bar{v}_{s-1}^{i}\right) E_{12} \\
\vdots \sum_{i=0, i \neq j-1}^{s-\lambda} \\
\bar{v}_{\lambda}^{j-1} E_{11}+\left(\sum_{i=0, i \neq j-1}^{i}\right) E_{12} \\
\bar{v}_{s-\lambda}^{j-1} E_{11}+\left(\sum_{s-1}^{i}\right) E_{12} \\
E_{11}+(s-1) E_{12} \\
\bar{v}_{1}^{j-1}\left(E_{11}-E_{12}\right) \\
\bar{v}_{s-1}^{j-1}\left(E_{11}-E_{12}\right) \\
\vdots \\
\bar{v}_{\lambda}^{j-1}\left(E_{11}-E_{12}\right) \\
\bar{v}_{s-\lambda}^{j-1}\left(E_{11}-E_{12}\right)
\end{array}\right]
$$

Then

$\left(\operatorname{diag}\left[I_{q_{0}}, M_{s} \otimes I_{q}\right]\right)^{H} E\left(\operatorname{diag}\left[I_{q_{0}}, M_{s} \otimes I_{k}\right]\right)$

$$
=\left[\begin{array}{c|c}
E_{00} & Z Z \\
\hline \sqrt{s} E_{10} & \\
0 & \left(M_{s} \otimes I_{q}\right)^{H} E_{1}\left(M_{s} \otimes I_{k}\right) \\
\vdots & \\
0 &
\end{array}\right]
$$

where

$$
\begin{gathered}
Z Z=\left[\begin{array}{cccc}
\sqrt{s} E_{01} & \frac{1}{\sqrt{s}} \sum_{i=0}^{s-1} \bar{v}_{1}^{i} E_{01} & \cdots & \frac{1}{\sqrt{s}} \sum_{i=0}^{s-1} \bar{v}_{s-\lambda}^{i} E_{01}
\end{array}\right] \\
=\left[\begin{array}{llll}
\sqrt{s} E_{01} & 0 & \cdots & 0
\end{array}\right]
\end{gathered}
$$

By equation (15), it follows that

$$
\begin{array}{r}
\left(M_{s} \otimes I_{q}\right)^{H} E_{1}\left(M_{s} \otimes I_{k}\right) \\
=\operatorname{diag}\left[E_{11}+(s-1) E_{12}, \quad E_{11}-E_{12}, \cdots\right. \\
\left.E_{11}-E_{12}\right]
\end{array}
$$


Thus,

$$
\begin{aligned}
& T_{R}^{T}\left(q_{0}, q, s\right) E T_{R}\left(k_{0}, k, s\right) \\
= & \left(\operatorname{diag}\left[I_{q_{0}}, U_{s} \otimes I_{q}\right]\right)^{H}\left[\left(\operatorname{diag}\left[I_{q_{0}}, M_{s} \otimes I_{q}\right]\right)^{H} E\right. \\
& \left.\times \operatorname{diag}\left[I_{k_{0}}, M_{s} \otimes I_{k}\right]\right] \operatorname{diag}\left[I_{k_{0}}, U_{s} \otimes I_{k}\right] \\
= & \operatorname{diag}\left[E_{p}, E_{m}, \cdots, E_{m}\right]
\end{aligned}
$$

For the case of $s$ even, the proof is similar.

The following notations will be used in the sequel.

$$
\begin{aligned}
& A_{p}=\left[\begin{array}{cl}
A_{11} & \sqrt{N-1} A_{12} \\
\sqrt{N-1} A_{12} & A_{11}+(N-2) A_{12}
\end{array}\right] \\
& B_{p}=\left[\begin{array}{c}
\sqrt{N-1} B_{12} \\
B_{11}+(N-2) B_{12}
\end{array}\right] \\
& C_{p}=\left[\begin{array}{ll}
\sqrt{N-1} C_{12} & C_{11}+(N-1) C_{12}
\end{array}\right] \\
& B_{p_{0}}=\left[\begin{array}{c}
B_{11} \\
\sqrt{N-1} B_{12}
\end{array}\right] \\
& C_{p_{0}}=\left[C_{11} \sqrt{N-1} C_{12}\right] \\
& G_{p}=\left[\begin{array}{cc}
G_{11} & \sqrt{N-1} G_{12} \\
\sqrt{N-1} G_{12} & G_{11}+(N-2) G_{12}
\end{array}\right] \\
& H_{p}=\left[\begin{array}{cc}
H_{11} & \sqrt{N-1} H_{12} \\
\sqrt{N-1} H_{12} & H_{11}+(N-2) H_{12}
\end{array}\right] \\
& A_{p 1}=A_{11}+(N-1) A_{12} \\
& B_{p 1}=(N+1)\left(B_{11}+(N-1) B_{12}\right) \text {. } \\
& C_{p 1}=(N+1)\left(C_{11}+(N+1) C_{12}\right) \\
& G_{p 1}=G_{11}+(N-1) G_{12} \\
& H_{p 1}=H_{11}+(N-1) H_{12} \\
& A_{m}=A_{11}-A_{12}, \quad B_{m}=B_{11}-B_{12} \\
& C_{m}=C_{11}-C_{12}, \quad G_{m}=G_{11}-G_{12} \\
& H_{m}=H_{11}-H_{12}
\end{aligned}
$$

\section{Reliable controller design}

In this section, we consider the reliable control problem for the linear system $\boldsymbol{\Sigma}$ of $(1-3)$ in case of sensor and actuator outages in the first subsystem. First, we review some results in [7] on the reliable control of general linear systems. Note that in Theorems 4.1 and 4.2 of [7], instead of AREs (Algebraic Riccati Equalities), only ARI (Algebraic Riccati In-qualities) are required. We summarize Theorems 4.1 and 4.2 of [7] as the following theorem. Denote

$$
\begin{array}{r}
\Delta_{x} \triangleq A^{T} X+X A-X B_{0} B_{0}^{T} X+\frac{1}{\gamma^{2}} X G G^{T} X \\
+H^{T} H+\gamma^{2} C_{1}^{T} C_{1} \\
\Delta_{y} \triangleq A Y+Y A^{T}-Y C_{0}^{T} C_{0} Y+\frac{1}{\gamma^{2}} Y H^{T} H Y \\
+G G^{T}+\gamma^{2} B_{1} B_{1}^{T}
\end{array}
$$

Theorem 1: [7] Given the linear system $\Sigma$ in $(1-3)$, suppose that $(A, H)$ is a detectable pair, and $0 \leq X \in$ $\mathbf{R}^{n N \times n N}$ and $0<Y \in \mathbf{R}^{n N \times n N}$ satisfy the AREs

$$
\begin{aligned}
& \Delta_{x}=0 \\
& \Delta_{y}=0
\end{aligned}
$$

respectively, with $\sigma_{\max }\{Y X\}<\gamma^{2}$ and $A+B K+$ $G_{+} K_{d_{+}}$and $A+B K+G_{+} K_{d_{+}}-L C$ Hurwitz where $\sigma(\cdot)$ is the singular value of a matrix and

$$
\begin{aligned}
K & =-B^{T} X \\
G_{+} & =\left[G \gamma B_{1}\right] \\
K_{d_{+}} & =\frac{1}{\gamma^{2}} G_{+}^{T} X \\
L & =\left(I-\gamma^{2} Y X\right)^{-1} Y C^{T} .
\end{aligned}
$$

Then the controller in (10) and (11) with

$$
\hat{w}=K_{d_{+}} \xi
$$

asymptotically stablizes the closed-loop system, and the $H_{\infty}$ norm of the closed-loop transfer function matrix from $\left[w^{T}, w_{0}^{T}\right]^{T}$ to $z$ is bounded by some prescribed $\gamma>0$ when all sensors and actuators are operational as well as when the sensors and actuators of one, but only one, prescribed subsystem experience outages.

Note that the orders of the two AREs in (18) and (19) are $n N \times n N$, and this poses enomous computation burden on the design process especially when $N$, the number of subsystems, is large. But the system $\Sigma$ in (1 - 3) under consideration has very strong symmetry. We show in this section that the this high-order problem of the two AREs is equivalent to four AREs of much lower dimensions: two of order $n \times n$ and two of order $2 n \times 2 n$. This reduces greatly the computational complexity of this reliable control problem. Denote

$$
\begin{gathered}
\Delta_{x p} \triangleq A_{p}^{T} X_{p}+X_{p} A_{p}-X_{p} B_{p} B_{p}^{T} X_{p} \\
+\frac{1}{\gamma^{2}} X_{p} G_{p} G_{p}^{T} X_{p}+H_{p}^{T} H_{p}+\gamma^{2} C_{p 0}^{T} C_{p 0} \\
\Delta_{x m} \triangleq A_{m}^{T} X_{m}+X_{m} A_{m}-X_{m} B_{m} B_{m}^{T} X_{m} \\
+\frac{1}{\gamma^{2}} X_{m} G_{m} G_{m}^{T} X_{m}+H_{m}^{T} H_{m} \\
\Delta_{y p} \triangleq A_{p}^{T} Y_{p}+Y_{p} A_{p}-Y_{p} C_{p} C_{p}^{T} Y_{p} \\
+\frac{1}{\gamma^{2}} Y_{p} H_{p} H_{p}^{T} Y_{p}+G_{p} G_{p}^{T}+\gamma^{2} B_{p 0} B_{p 0}^{T} \\
\Delta_{y m} \triangleq A_{m}^{T} Y_{m}+Y_{m} A_{m}-Y_{m} C_{m} C_{m}^{T} Y_{m} \\
+\frac{1}{\gamma^{2}} Y_{m} H_{m} H_{m}^{T} Y_{m} G_{m} G_{m}^{T}
\end{gathered}
$$

Theorem 2: Suppose that the pairs $\left(A_{p 1}, H_{p 1}\right)$ and $\left(A_{m}, H_{m}\right)$ are detectable, and $\gamma$ is a positive constant. Suppose also that the following hold. 
1. There exist $0 \leq X_{p} \in \mathbf{R}^{2 n \times 2 n}$ and $0 \leq X_{m} \in$ $\mathbf{R}^{n \times n}$ such that

$$
\begin{aligned}
\Delta_{x p} & =0 \\
\Delta_{x m} & =0 .
\end{aligned}
$$

2. There exist $0 \leq Y_{p} \in \mathbf{R}^{2 n \times 2 n}$ and $0 \leq Y_{m} \in$ $\mathbf{R}^{n \times n}$ such that

$$
\begin{aligned}
\Delta_{y p} & =0 \\
\Delta_{y m} & =0 .
\end{aligned}
$$

3. (a) The matrices $A_{p}-B_{p} B_{p}^{T} X_{p}+1 / \gamma^{2} G_{p} G_{p}^{T} X_{p}$ and $A_{m}-B_{m} B_{m}^{T} X_{m}+1 / \gamma^{2} G_{m} G_{m}^{T}$ are Hurwitz; (b)

$$
\max \left\{\sigma_{\max }\left(Y_{p} X_{p}\right), \quad \sigma_{\max }\left(Y_{m} X_{m}\right)\right\}<\gamma^{2} ;
$$

(c) the two closed-loop matrices $A_{p}-B_{p} B_{p}^{T} X_{p}$ $+1 / \gamma^{2} G_{p} G_{p}^{T} X_{p} \quad-\quad-\quad L_{p} \bar{C}_{p}$ and $A_{m}-B_{m} B_{m}^{T} X_{m}+1 / \gamma^{2} G_{m} G_{m}^{T} X_{m}-L_{m} C_{m}$ are Hurwitz, where

$$
\begin{aligned}
\bar{C}_{p} & =\left[\begin{array}{cc}
C_{11} & \sqrt{N-1} C_{12} \\
\sqrt{N-1} C_{12} & C_{11}+(N-1) C_{12}
\end{array}\right] \\
L_{p} & =\left(I-\gamma^{2} Y_{p} X_{p}\right)^{-1} Y_{p} \bar{C}_{p}^{T} \\
L_{m} & =\left(I-\gamma^{2} Y_{m} X_{m}\right)^{-1} Y_{m} C_{m}^{T} .
\end{aligned}
$$

Then there exists a controller of the form (7) and (8) such that the resulting closed-loop system is asymptotically stable and has the $H_{\infty}$-norm bound not greater than $\gamma$ when all sensors and actuators are operational as well as when the sensors and actuators of the first subsystem experience outages.

Furthermore, the construction of the controller is given as follows. Partition $X_{p}$ and $Y_{p}$ into $n \times n$ blocks

$$
X_{p}=\left[\begin{array}{ll}
X_{p 00} & X_{p 01} \\
X_{p 10} & X_{p 11}
\end{array}\right], \quad Y_{p}=\left[\begin{array}{cc}
Y_{p 00} & Y_{p 01} \\
Y_{p 10} & Y_{p 11}
\end{array}\right]
$$

and let

$$
\begin{aligned}
X= & {\left[\begin{array}{ccccc}
X_{00} & X_{01} & \cdots & X_{01} & X_{01} \\
X_{10} & X_{11} & \cdots & X_{12} & X_{12} \\
\vdots & \vdots & \ddots & \vdots & \vdots \\
X_{10} & X_{12} & \cdots & X_{11} & X_{12} \\
X_{10} & X_{12} & \cdots & X_{12} & X_{11}
\end{array}\right] } \\
Y= & {\left[\begin{array}{ccccc}
Y_{00} & Y_{01} & \cdots & Y_{01} & Y_{01} \\
Y_{10} & Y_{11} & \cdots & Y_{12} & Y_{12} \\
\vdots & \vdots & \ddots & \vdots & \vdots \\
Y_{10} & Y_{12} & \cdots & Y_{11} & Y_{12} \\
Y_{10} & Y_{12} & \cdots & Y_{12} & Y_{11}
\end{array}\right] }
\end{aligned}
$$

where

$$
X_{00}=X_{p 00}, \quad X_{01}=1 / \sqrt{N-1} X_{p 01}
$$

$$
\begin{aligned}
X_{10} & =1 / \sqrt{N-1} X_{p 10} \\
X_{11} & =1 /(N-1)\left[X_{p 11}+(N-2) X_{m}\right] \\
X_{12} & =1 /(N-1)\left[X_{p 11}-X_{m}\right] \\
Y_{00} & =Y_{p 00}, \quad Y_{01}=1 / \sqrt{N-1} Y_{p 01} \\
Y_{10} & =1 / \sqrt{N-1} Y_{p 10} \\
Y_{11} & =1 /(N-1)\left[Y_{p 11}+(N-2) Y_{m}\right] \\
Y_{12} & =1 /(N-1)\left[Y_{p 11}-Y_{m}\right] .
\end{aligned}
$$

Then, the controller of equation (7) and (8) is given by $(20-23)$ of Theorem 1.

The following lemma will be used in the proof of Theorem 2.

Lemma 2: Under the assumptions of Theorem 2, the following hold:

1.

$$
\begin{aligned}
& \Delta_{x}=0 \\
& \Delta_{y}=0
\end{aligned}
$$

2. the matrix $A+B K+G_{+} K_{d_{+}}=A-B_{0} B_{0}^{T} X+$ $\frac{1}{\gamma^{2}} G G^{T} X$ is Hurwitz;

3. $\sigma_{\max }\{Y X\}<\gamma^{2}$;

4. the controller given by (7) and (8) with (20 - 23) is open-loop stable.

Proof: 1. By Lemma 1 and the orthogonality of the matrix $T_{R}(p, q, s)$, we have

$$
\begin{aligned}
& T_{R}^{T}(n, n, N-1) A^{T} X T_{R}(n, n, N-1) \\
= & T_{R}^{T}(n, n, N-1) A^{T} T_{R}(n, n, N-1) \\
& \quad \times T_{R}^{T}(n, n, N-1) X T_{R}(n, n, N-1) \\
= & \operatorname{diag}\left[A_{p}, A_{m}, \cdots, A_{m}\right] \operatorname{diag}\left[X_{p}, X_{m}, \cdots, X_{m}\right] \\
= & \operatorname{diag}\left[A_{p} X_{p}, A_{m} X_{m}, \cdots, A_{m} X_{m}\right] .
\end{aligned}
$$

Similarly,

$$
\begin{gathered}
T_{R}^{T}(n, n, N-1) X A T_{R}(n, n, N-1) \\
=\operatorname{diag}\left[A_{p} X_{p}, A_{m} X_{m}, \cdots, A_{m} X_{m}\right] \\
T_{R}^{T}(n, n, N-1) X B_{0} B_{0}^{T} X T_{R}(n, n, N-1) \\
=T_{R}^{T}(n, n, N-1) X T_{R}(n, n, N-1) \\
\times T_{R}^{T}(n, n, N-1) B_{0} T_{R}(m, m, N-1) \\
\times T_{R}^{T}(m, m, N-1) B_{0}^{T} T_{R}(n, n, N-1) \\
\times T_{R}^{T}(n, n, N-1) X T_{R}(n, n, N-1) \\
=\operatorname{diag}\left[X_{p} B_{p} B_{p}^{T} X_{p}, X_{m} B_{m} B_{m}^{T} X_{m}, \cdots,\right. \\
\left.X_{m} B_{m} B_{m}^{T} X_{m}\right]
\end{gathered}
$$




$$
\begin{aligned}
& T_{R}^{T}(n, n, N-1) X G G^{T} X T_{R}(n, n, N-1) \\
= & \operatorname{diag}\left[X_{p} G_{p} G_{p}^{T} X_{p}, X_{m} G_{m} G_{m}^{T} X_{m}, \cdots,\right. \\
& \left.X_{m} G_{m} G_{m}^{T} X_{m}\right] \\
& T_{R}^{T}(n, n, N-1) H^{T} H T_{R}(n, n, N-1) \\
= & \operatorname{diag}\left[H_{p}^{T} H_{p}, H_{m}^{T} H_{m}, \cdots, H_{m}^{T} H_{m}\right] \\
& T_{R}^{T}(n, n, N-1) C_{1}^{T} C_{1} T_{R}(n, n, N-1) \\
= & \operatorname{diag}\left[C_{p 0}^{T} C_{p 0}, 0, \cdots, 0\right] .
\end{aligned}
$$

From equalities (29) and (30), it follows

$$
\begin{aligned}
& T_{R}^{T}(n, n, N-1) \Delta_{x} T_{R}(n, n, N-1) \\
& \quad=\operatorname{diag}\left[\Delta_{x p}, \Delta_{x m}, \cdots, \Delta_{x m}\right]=0
\end{aligned}
$$

By assumption 1 in Theorem 2, $\Delta_{x p}=0$ and $\Delta_{x m}=0$. Thus, the equality (37) holds. The proof for equality (38) is similar.

Conclusions 2, 3 and 4 follow from the assumption 3 of Theorem 2 and the similar arguments as above.

Proof of Theorem 2: Note that $C=C_{0}+C_{1}, B=$ $B_{0}+B_{1}$, then the proof is completed by using Theorem 1 and Lemma 2.

Remark 1: Theorem 2 presents a reliable controller design procedure for symmetric composite systems in terms of the solutions of the algebraic Reccati equations (AREs). But the order of these AREs are much lower than that of Theorem 1. The resulting closedloop system is reliable with respect to the outage of sensors and actuators of a prescribed sybsystems (the first subsystem, in this case). This result is also different from that given in [6] [8] where the solution of the ARE for a symmetric composite system is constructed from the solutions of two AREs of the same order as the subsystem. When only sensor outages, or only actuator outages are considered, simpler design procedures can be obtained from Theorem 2 and its proof. The details are omitted.

Remark 2: It should also be noted that the condition under which the pair $\left(A_{p}, B_{p}\right)$ is stabilizable and the pair $\left(A_{p}, C_{p}\right)$ is detectable, is a necessary condition for the equation (29) and (31) to have positive definite solutions. This condition requires that the unstable modes of the prescribed subsystem be controlled or detected by other subsystem inputs or outputs through the coupling between the subsystems. When the matrix $A_{m}=A_{11}-A_{12}$ is unstable, it can be shown by a method similar to that in the proof of Theorem 2, that the reliable controller design is impossible for the case in which the sensors and actuators of any two subsystems are susceptible to outages.

\section{Conclusion}

This paper treats the reliable control problems for symmetric composite systems composed of several identical subsystems. By taking advantage of the symmetric structure of the systems, a reliable controller design procedure is presented in terms of the solutions to the algebraic Riccati equations with lower-order. The resulting control systems are reliable in that they provide guaranteed asymptotic stability and $H_{\infty}$ performance when all sensors and actuators are operational as well as when the sensors or actuators of a prescribed subsystem experience outages.

\section{References}

[1] J. Lunze, "Dynamics of strongly coupled symmetric composite systems", Int. J. Control, 44, 1657$1670,1986$.

[2] J. Lunze, "Stability analysis of large scale systems composed of strongly coupled similar subsystems", Automatica, 25, 561-570, 1989.

[3] J. V. Medanic, "Design of reliable controllers using redundant control elements", Proceedings of the 1993 ACC, San Francisco, 3, 3130-3134, 1993.

[4] M. H. Shor, W. R. Perkins, and J. V. Medanic, "Design of reliable decentralized controllers: A unified continuous/discrete formulation", Int. J. Control, $\mathbf{5 6}$, 943-965, 1992.

[5] D. D. Siljak, "Reliable control using multiple control systems", Int. J. Control, 31, 303-329, 1980.

[6] M. K. Sundareshan and R. M. Elbanna, "Qualitative analysis and decentralized controller synthesis for a class of large scale systems with symmetrically interconnected subsystems", Automatica, 27, 383-388, 1991.

[7] R. J. Veillette, J. V. Medanic and W. R. Perkins, "Design of reliable control systems", IEEE Trans. Automat. Control, 37, 290-304, 1992.

[8] G. H. Yang and S. Y. Zhang, "Stabilizing controllers for uncertain symmetric composite systems", Automatica, 31, 337-340, 1995. 